\title{
Unsteady/Steady Hydromagnetic Convective Flow between Two Vertical Walls in the Presence of Variable Thermal Conductivity
}

\author{
M. M. Hamza, ${ }^{1}$ I. G. Usman, ${ }^{2}$ and A. Sule ${ }^{2}$ \\ ${ }^{1}$ Department of Mathematics, Usmanu Danfodiyo University, PMB 2346, Sokoto, Nigeria \\ ${ }^{2}$ Department of Mathematics, Zamfara State College of Education, PMB 1002, Maru, Nigeria \\ Correspondence should be addressed to M. M. Hamza; hmbtamb@yahoo.com
}

Received 16 September 2014; Revised 25 March 2015; Accepted 1 April 2015

Academic Editor: Miguel Onorato

Copyright (C) 2015 M. M. Hamza et al. This is an open access article distributed under the Creative Commons Attribution License, which permits unrestricted use, distribution, and reproduction in any medium, provided the original work is properly cited.

\begin{abstract}
Unsteady as well as steady natural convection flow in a vertical channel in the presence of uniform magnetic field applied normal to the flow region and temperature dependent variable thermal conductivity is studied. The nonlinear partial differential equations governing the flow have been solved numerically using unconditionally stable and convergent semi-implicit finite difference scheme. For steady case, approximate solutions have been derived for velocity, temperature, skin friction, and the rate of heat transfer using perturbation series method. Results of the computations for velocity, temperature, skin friction, and the rate of heat transfer are presented graphically and discussed quantitatively for various parameters embedded in the problem. An excellent agreement was found during the numerical computations between the steady-state approximate solutions and unsteady numerical solutions at steady-state time. In addition, comparison with previously published work is performed and the results agree well.
\end{abstract}

\section{Introduction}

In recent years, the interest in the study of hydromagnetic flow in a channel region has been growing rapidly because of its extensive engineering applications. The experimental investigation of modern MHD flow in a laboratory was first carried out by [1]. This study provided the basic knowledge for the development of many MHD devices, such as MHD pumps, MHD generators, brakes, flow meters, plasma studies, and geothermal energy extraction. Unsteady free convection heat transfer with MHD effects in a channel region can be found in [2]. Unsteady hydromagnetic flows in rotating systems have been studied by [3-9]. An exact solution for unsteady hydromagnetic free convection flow with constant heat flux is to be found in [10]. All the above mentioned studies assumed the thermal conductivity of the fluid to be constant. However, it is known that the fluid physical properties may change significantly with temperature changes. To accurately predict the flow behavior and heat transfer rate, it is necessary to take into account the variation of thermal conductivity with temperature (see [11]). Thermal properties, particularly thermal conductivity and diffusivity, are essential materials parameters of bedrock controlling the heat transfer and temperature increases in the vicinity of repository. There has been considerable published work dealing with steady flow with variable thermal conductivity (see [12-18]). Recently (see [19]) studied steady MHD flow with variable thermal conductivity over an inclined radiative isothermal permeable surface.

To the best of our knowledge, the problem of unsteady/ steady hydromagnetic convective flow between two vertical walls heated symmetrically/asymmetrically in the presence of variable thermal conductivity has not been studied. The present paper is committed to study unsteady as well as steady natural convection flow of a viscous, incompressible fluid between two parallel vertical walls in the presence of transverse magnetic field and temperature dependent variable thermal conductivity when convection between the vertical parallel walls is set up by a change in the temperature of the walls compared to the fluid temperature.

\section{Governing Equations}

Consider the unsteady natural convection flow of viscous, incompressible, and electrically conducting fluid between 


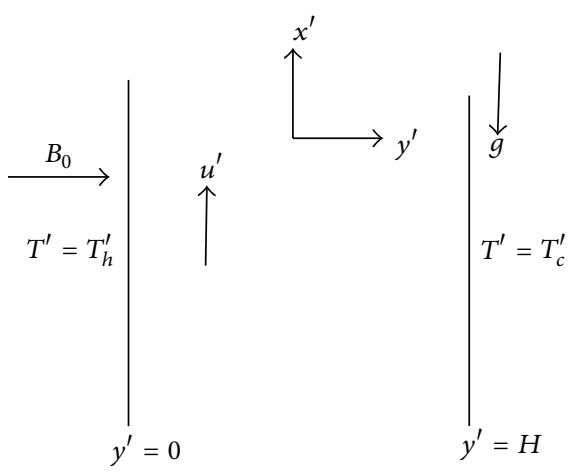

FIGURE 1: Geometry of the problem.

two vertical walls in the presence of a transversely imposed magnetic field of strength $B_{0}$. Initially, it is assumed that both the fluid and the walls are at rest and at the same temperature $T_{m}^{\prime}$. At time $t^{\prime}>0$, the temperature of the walls $y^{\prime}=0$ and $y^{\prime}=H$ is instantaneously raised or lowered to $T_{h}^{\prime}$ and $T_{c}^{\prime}$, respectively, such that $T_{h}^{\prime}>T_{c}^{\prime}$ which is thereafter maintained constant. We chose a Cartesian coordinate system with $x^{\prime}$ axis along the upward direction and the $y^{\prime}$ axis normal to it as shown in Figure 1. Thermal conductivity $\left(k_{f}\right)$ of the fluid is assumed to vary as a linear function of temperature in the form (see [20]), $k_{f}=k_{m}\left[1+\delta\left(T^{\prime}-T_{m}^{\prime}\right)\right]$, where $k_{m}$ is the fluid free stream thermal conductivity and $\delta$ is a constant depending on the nature of the fluid, where $\delta>0$ for fluids such as water and air, while $\delta<0$ for fluids such as lubrication oils (see [20]). The governing equations under Boussinesq's approximation can be written as

$$
\begin{aligned}
& \frac{\partial u^{\prime}}{\partial t^{\prime}}=\nu \frac{\partial^{2} u^{\prime}}{\partial y^{\prime 2}}+g \beta\left(T^{\prime}-T_{m}^{\prime}\right)-\frac{\sigma B_{0}^{2} u^{\prime}}{\rho} \\
& \frac{\partial T^{\prime}}{\partial t^{\prime}}=\frac{1}{\rho c_{p}} \frac{\partial}{\partial y^{\prime}}\left[k_{f} \frac{\partial T^{\prime}}{\partial y^{\prime}}\right] .
\end{aligned}
$$

The initial and boundary conditions for the present problem are

$$
\begin{aligned}
& t^{\prime} \leq 0: u^{\prime}=0, T^{\prime}=T_{m}^{\prime}, \text { for } 0 \leq y^{\prime} \leq H, \\
& t^{\prime}>0: u^{\prime}=0, T^{\prime}=T_{h}^{\prime} \text { at } y^{\prime}=0, \\
& u^{\prime}=0, T^{\prime}=T_{c}^{\prime} \text { at } y^{\prime}=H,
\end{aligned}
$$

where $\beta$ is the coefficient of the thermal expansion, $v$ is the kinematic viscosity, $g$ is the gravitational force, $\sigma$ is the conductivity of the fluid, $B_{0}$ is the electromagnetic induction, $\rho$ is the density of the fluid, and $c_{p}$ is the specific heat at constant pressure.
In order to solve (1) to (2), we employ the following dimensionless parameters:

$$
\begin{aligned}
y & =\frac{y^{\prime}}{H}, \\
t & =\frac{t^{\prime} v}{H^{2}}, \\
U & =\frac{u^{\prime} v}{g \beta\left(T_{h}^{\prime}-T_{m}^{\prime}\right) H^{2}}, \\
M^{2} & =\frac{\sigma B_{0}^{2} H^{2}}{\nu \rho}, \\
\operatorname{Pr} & =\frac{\nu \rho c_{p}}{k_{m}}, \\
\theta & =\frac{T^{\prime}-T_{m}^{\prime}}{T_{h}^{\prime}-T_{m}^{\prime}}, \\
R & =\frac{T_{c}^{\prime}-T_{m}^{\prime}}{T_{h}^{\prime}-T_{m}^{\prime}}, \\
\lambda & =\delta\left(T_{h}^{\prime}-T_{m}^{\prime}\right) .
\end{aligned}
$$

Using (3), (1) to (2) can take the following form:

$$
\begin{aligned}
\frac{\partial U}{\partial t} & =\frac{\partial^{2} U}{\partial y^{2}}+\theta-M^{2} U, \\
\operatorname{Pr} \frac{\partial \theta}{\partial t} & =(1+\lambda \theta) \frac{\partial^{2} \theta}{\partial y^{2}}+\lambda\left(\frac{\partial \theta}{\partial y}\right)^{2} .
\end{aligned}
$$

The initial and boundary conditions in dimensionless form are

$$
\begin{aligned}
& t \leq 0: U=0, \theta=0,0 \leq y \leq 1, \\
& t>0: U=0, \theta=1, \text { at } y=0, \\
& U=0, \theta=R, \text { at } y=1 .
\end{aligned}
$$

\section{Approximate Solutions}

The approximated solutions played an important role in validating and exploring computer routes of complicated problems. Therefore, we reduce the governing equations of this problem due to its nonlinearity into a form that can be solved analytically. By setting $\partial u / \partial t=0$, and $\partial \theta / \partial t=0$ into (4), we get

$$
\begin{aligned}
\frac{d^{2} U}{d y^{2}}-M^{2} U & =-\theta, \\
(1+\lambda \theta) \frac{d^{2} \theta}{d y^{2}}+\lambda\left(\frac{d \theta}{d y}\right)^{2} & =0 .
\end{aligned}
$$

The boundary conditions are

$$
\begin{aligned}
& U=0, \theta=1, \text { at } y=0 \\
& U=0, \theta=R, \text { at } y=1 .
\end{aligned}
$$


In order to construct an approximate solution to (6) and (7) subject to (8), we employed a regular perturbation method by taking a power series expansion in the variable thermal conductivity parameter $\lambda$ :

$$
\begin{gathered}
U=U_{0}+\lambda U_{1}, \\
\theta=\theta_{0}+\lambda \theta_{1} .
\end{gathered}
$$

Substituting (9) into (6) to (8), the solution of the governing equations are obtained as

$$
\begin{aligned}
U & =\frac{1}{M^{2}}[1+(R-1) y-\cosh (M y) \\
& \left.+\left(k_{2}-k_{3}\right) \sinh (M y)\right]+\lambda[A \cosh (M y) \\
& \left.+B \sinh (M y)+k_{4} y^{3}+k_{5} y^{2}+k_{6} y+k_{7}\right], \\
\theta & =1+(R-1) y+\lambda\left[k_{1} y-(R-1) y^{2}\right. \\
& \left.-(R-1)^{2} \frac{y^{3}}{3}\right] .
\end{aligned}
$$

Using (10), we write the steady-state skin friction and rate of heat transfer on the boundaries as follows.

Steady-state skin friction on the boundary plates is

$$
\begin{aligned}
& \left.\frac{\partial U}{\partial y}\right|_{y=0}=\frac{1}{M^{2}}\left[(R-1)-M\left(k_{2}-k_{3}\right)\right]+\lambda\left[k_{6}\right. \\
& -B M] \\
& \left.\frac{\partial U}{\partial y}\right|_{y=1}=\frac{1}{M^{2}}[(R-1)-M \sinh (M) \\
& \left.-M\left(k_{2}-k_{3}\right) \cosh (M)\right]+\lambda[A M \sinh (M) \\
& \left.-B M \cosh (M)+3 k_{4}+2 k_{5}+k_{6}\right] .
\end{aligned}
$$

The steady-state rate of heat transfer on the boundary plates is

$$
\begin{aligned}
& \left.\frac{\partial \theta}{\partial y}\right|_{y=0}=(R-1)+\lambda k_{1} \\
& \left.\frac{\partial \theta}{\partial y}\right|_{y=1}=(R-1)+\lambda\left[k_{1}-2(R-1)-(R-1)^{2}\right],
\end{aligned}
$$

where

$$
\begin{aligned}
& k_{1}=\frac{(R-1)^{2}+3(R-1)}{3}, \\
& k_{2}=\frac{\cosh (M)}{\sinh (M)}, \\
& k_{3}=\frac{R}{\sinh (M)},
\end{aligned}
$$

$$
\begin{aligned}
& k_{4}=-\frac{(R-1)^{2}}{3 M^{2}}, \\
& k_{5}=-\frac{(R-1)}{M^{2}}, \\
& k_{6}=\frac{k_{1}+6 k_{4}}{M^{2}}, \\
& k_{7}=\frac{2 k_{5}}{M^{2}}, \quad A=-k_{7}, \\
& B=\frac{k_{7} \cosh (M)}{\sinh (M)}-\frac{\left(k_{4}+k_{5}+k_{6}+k_{7}\right)}{\sinh (M)} .
\end{aligned}
$$

\section{Numerical Solutions}

The complete forms of (4) are solved numerically using semiimplicit finite difference scheme. We used forward difference formulas for all time derivatives and approximate the spatial derivatives with central difference formula. The semi-implicit finite difference equations corresponding to (4) are as follows:

$$
\begin{aligned}
& -r_{1} U_{j-1}^{(N+1)}+\left(1+2 r_{1}\right) U_{j}^{(N+1)}-r_{1} U_{j+1}^{(N+1)} \\
& =r_{2} U_{j-1}^{(N)}+\left(1-2 r_{2}-M^{2} \Delta t\right) U_{j}^{(N)}+r_{2} U_{j+1}^{(N)} \\
& \quad+\Delta t \theta_{j}^{N} \\
& -r_{1} \theta_{j-1}^{(N+1)}+\left(\operatorname{Pr}+2 r_{1}\right) \theta_{j}^{(N+1)}-r_{1} \theta_{j+1}^{(N+1)} \\
& =\left(r_{2}+r_{3}\right) \theta_{j-1}^{(N)}+\left(\operatorname{Pr}-2 r_{2}-2 r_{3}\right) \theta_{j}^{(N)} \\
& \quad+\left(r_{2}+r_{3}\right) \theta_{j+1}^{(N)}+r_{4}\left(\theta_{j+1}^{(N)}-\theta_{j-1}^{(N)}\right)^{2},
\end{aligned}
$$

where $r_{1}=\xi \Delta t / \Delta y^{2}, r_{2}=(1-\xi) \Delta t / \Delta y^{2}, r_{3}=\lambda \Delta t \theta_{j}^{(N)} / \Delta y^{2}$, $r_{4}=\lambda \Delta t / 4 \Delta y^{2}$, and $0 \leq \xi \leq 1$. We chose $\xi=1$ so that we are free to choose larger time steps. The approximated solutions displayed in the previous section are used as a check on the accuracy and effectiveness of the numerical scheme. Also, in order to reconfirm accuracy of the scheme, the numerical results for velocity and temperature are compared with the approximated solutions. It has been found that numerical values of the velocity and temperature fields calculated from expressions (10) have matched very well with the numerical solutions obtained from expressions (14) at the steady-state time. See Figure 2 for the graph of the numerical solutions at steady-state and steady-state approximate solutions for velocity and temperature fields. Again, Figures 3 and 4 represent comparisons with the work of [21] when $\operatorname{Pr}=0.71, M=0$, and $\lambda=0$. It is clear that excellent agreement between the present numerical solutions and the approximated solutions of [21] exists.

\section{Results and Discussion}

The numerical results are obtained by solving (14) using the method described in the previous section for various values 


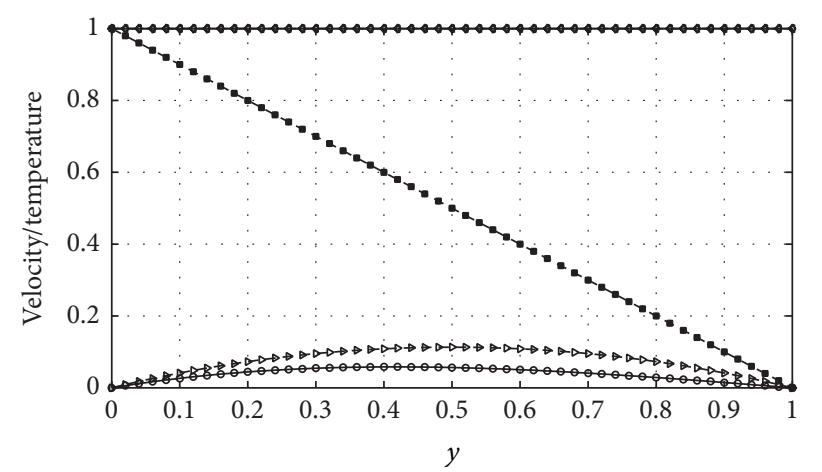

Num. soln. for vel. $R=0 \quad \ldots$ Num. soln. for temp. $R=0$

- Analyt. soln. for vel. $\mathrm{R}=0$ a Analyt. soln. for temp. $\mathrm{R}=0$

- - N Num. soln. for vel. $\mathrm{R}=1 \quad-\triangleleft-$ Num. soln. for temp. $R=1$

$\triangleright \quad$ Analyt. soln. for vel. $\mathrm{R}=1 \quad \diamond \quad$ Analyt. soln. for temp. $R=1$

FIgURE 2: Unsteady and steady-state solutions for velocity and temperature profiles.

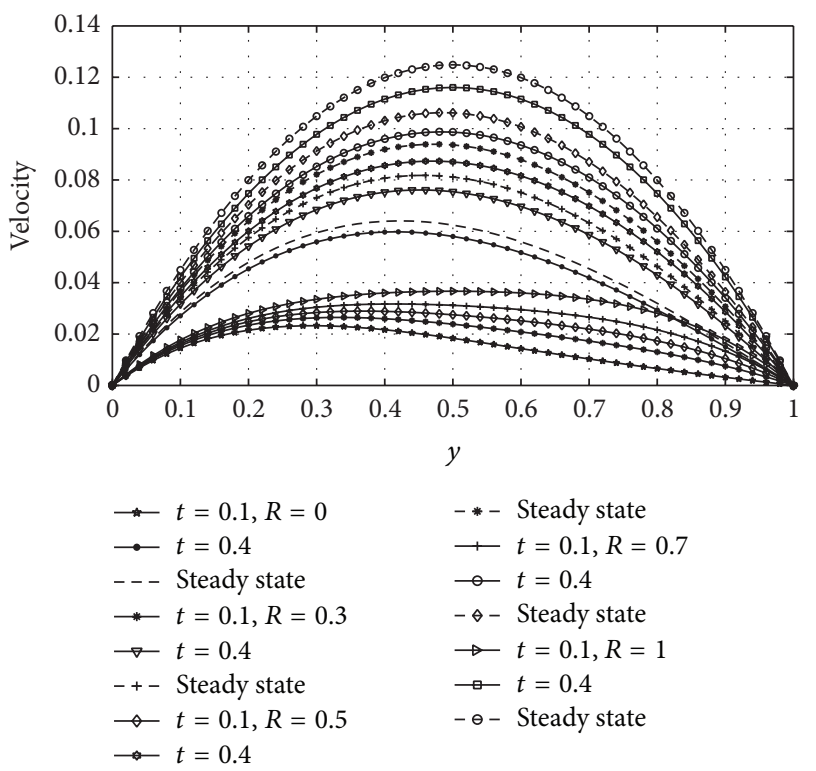

FIGURE 3: Unsteady and steady-state solutions for velocity profiles $M=0, \operatorname{Pr}=0.71$, and $\lambda=0$.

of physical parameters to describe the physics of the problem. The nondimensional parameters that govern the flow are the Prandtl number $(\mathrm{Pr})$, magnetic parameter $(M)$, variable thermal conductivity parameter $(\lambda)$, and buoyancy force distribution parameter $(R)$. The value of Prandtl number $(\mathrm{Pr})$ is taken as 1.0, which corresponds to electrolyte solution (see $[22,23])$. Results obtained are presented graphically for velocity, temperature, skin friction, and Nusselt number for various flow parameters.

Figures 5 and 6 illustrate the velocity profiles of the fluid for different values of magnetic parameter (M) and nondimensional time $(t)$ for asymmetric and symmetric case, respectively (i.e., $R=0$ and $R=1$ ). From Figure 5 it is observed that velocity of the fluid is maximum near

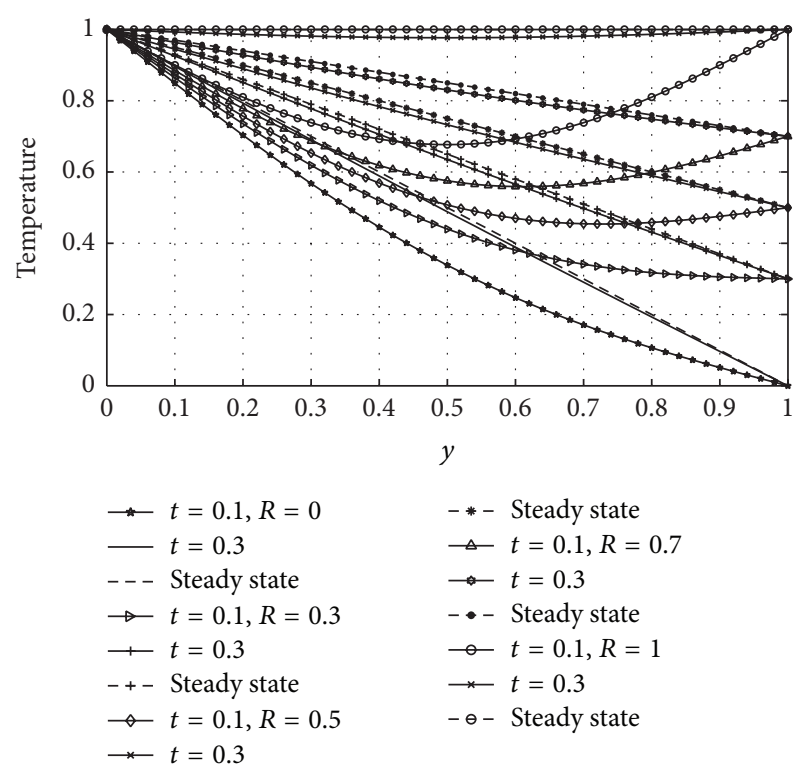

FIGURE 4: Unsteady and steady-state solutions for temperature profiles $M=0, \operatorname{Pr}=0.71$, and $\lambda=0$.

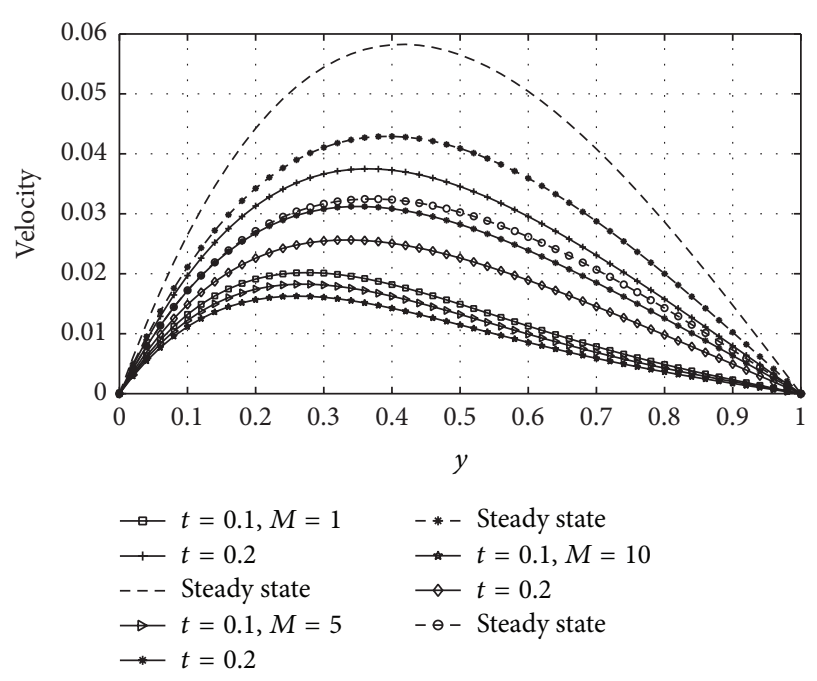

FIGURE 5: Unsteady and steady-state solutions for velocity profiles $R=0, \operatorname{Pr}=1$, and $\lambda=0.1$.

the heated wall $(y=0)$ and then gradually decreases as it moves towards the cooled wall $(y=1)$. It is clear from Figure 6 that symmetric flow about occurs between the walls for all considered values of $M$ and the nature of the figures is parabolic. In both Figures 5 and 6 it is noted that increasing $M$ decreases the velocity of the flow throughout the channel walls. The physical explanation of this behavior is that the presence of magnetic field produces a resistivity force (Lorentz force) similar to the drag force which retard the velocity.

Figures 7 and 8 , respectively, show the response of the fluid temperature to variation in the variable thermal conductivity parameter $(\lambda)$ and time $(t)$ for asymmetric and 


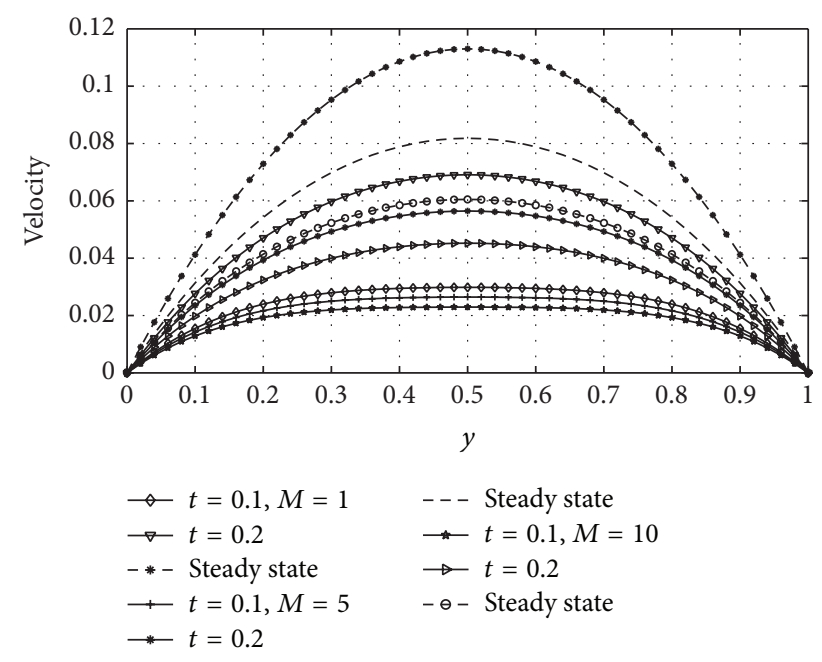

FIGURE 6: Unsteady and steady-state solutions for velocity profiles $R=1, \operatorname{Pr}=1$, and $\lambda=0.1$.

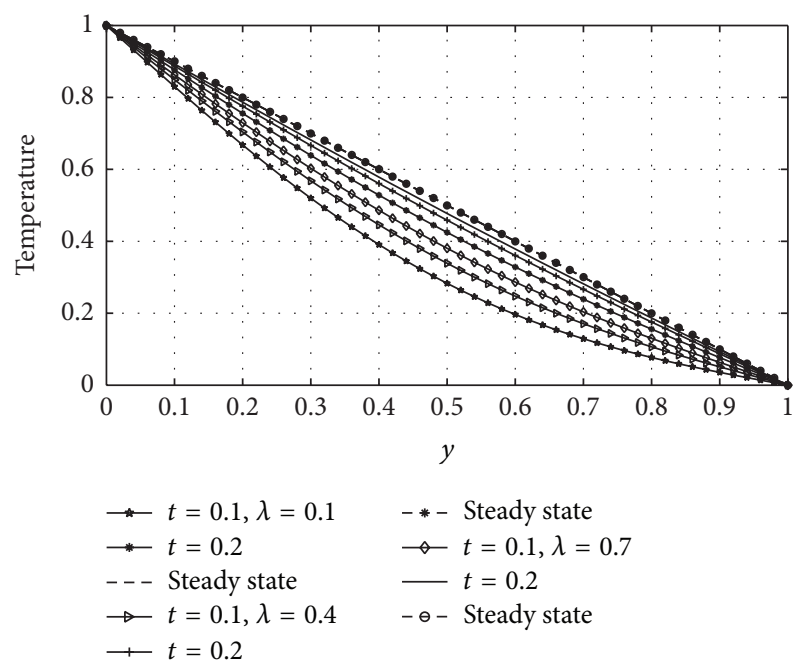

FIGURE 7: Unsteady and steady-state solutions for temperature profiles $R=0, \operatorname{Pr}=1$, and $M=1$.

symmetric case. The effect of variable thermal conductivity $(\lambda)$ and time $(t)$ on velocity profiles for asymmetric and symmetric case is shown in Figures 9 and 10, respectively. From Figures 7 to 10, it is observed that both temperature and velocity of the fluid increase with increasing $\lambda$ and $t$ until a steady-state condition is attained. This is physically true, since the relation $\lambda=\delta\left(T_{h}^{\prime}-T_{m}^{\prime}\right)$ indicates that mounting values of $\lambda$ increase the temperature difference between outside the plate and outside the boundary layer. As a result, heat is transferred rapidly from plate to fluid within the boundary layer. That is why both velocity and temperature profiles enlarge due to growing $\lambda$. It means that the velocity and the thermal boundary layer thickness rise for larger $\lambda$. From Figure 9, it is also seen that velocity of the fluid is maximum near the heated wall $(y=0)$ and then progressively decreases as it moves towards the cooled wall $(y=1)$. It is clear from

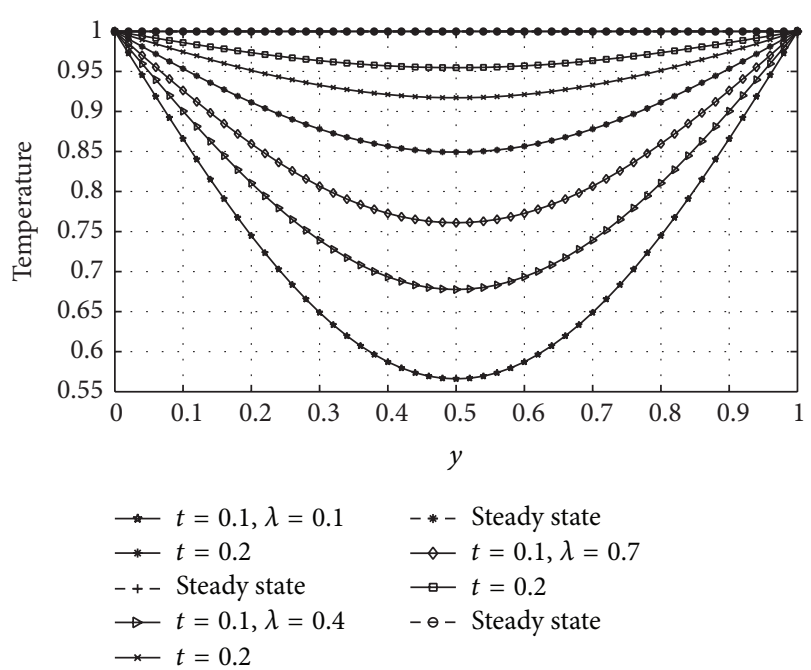

FIGURE 8: Unsteady and steady-state solutions for temperature profiles $R=1, \operatorname{Pr}=1$, and $M=1$.

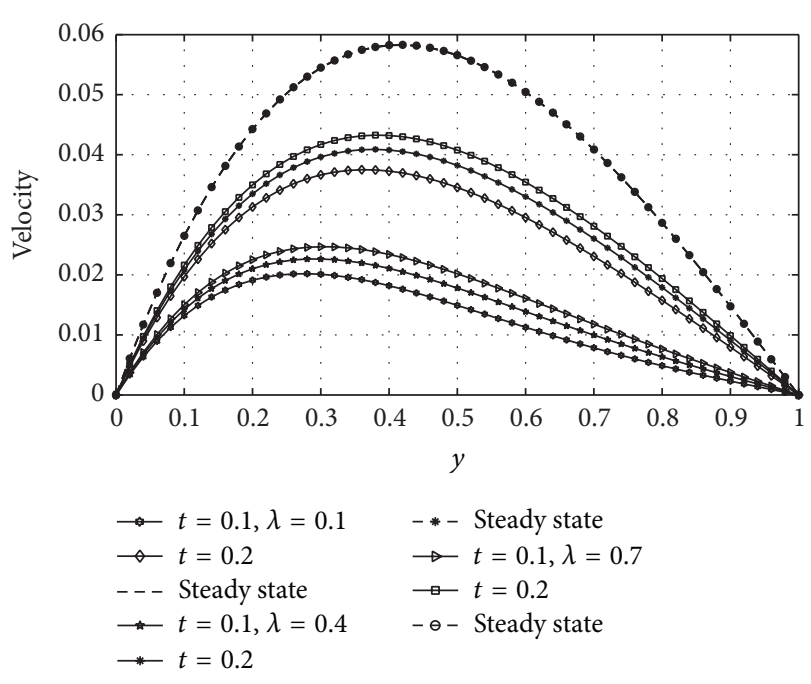

FIGURE 9: Unsteady and steady-state solutions for velocity profiles $R=0, \operatorname{Pr}=1$, and $M=1$.

Figures 8 and 10 that symmetric flow about occurs between the walls for all considered values of $\lambda$ and $t$ until a steadystate condition is achieved.

The rate of heat transfer (Nusselt number) dependence on $\lambda$ is illustrated in Figure 11 for symmetric case. Figures $11(\mathrm{a})$ and 11(b) represent the rate of heat transfer at the walls $y=0$ and $y=1$, respectively. Figure 11(b) reveals that the rate of heat transfer increases as $\lambda$ and $t$ increase until a steady-state condition is reached. A reverse effect is observed at the plate $y=0$; see Figure 11(a). The wall shear stress (skin friction) dependence on $\lambda$ for varying values of $t$ is illustrated in Figures 12(a) and 12(b) for symmetric case at the plates $y=0$ and $y=1$, respectively. From these figures, it is seen that skin friction increases with increase in $\lambda$ and $t$. The skin friction and Nusselt number dependence on $\lambda$ for varying values of $t$ is displayed in Figures 13(a) and 13(b), respectively, 


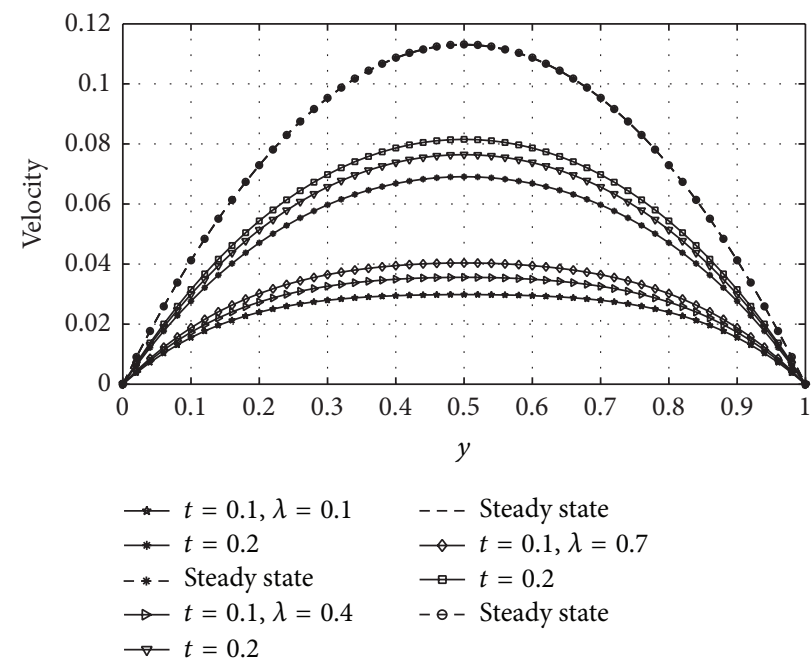

FIgURE 10: Unsteady and steady-state solutions for velocity profiles $R=1, \operatorname{Pr}=1$, and $M=1$.

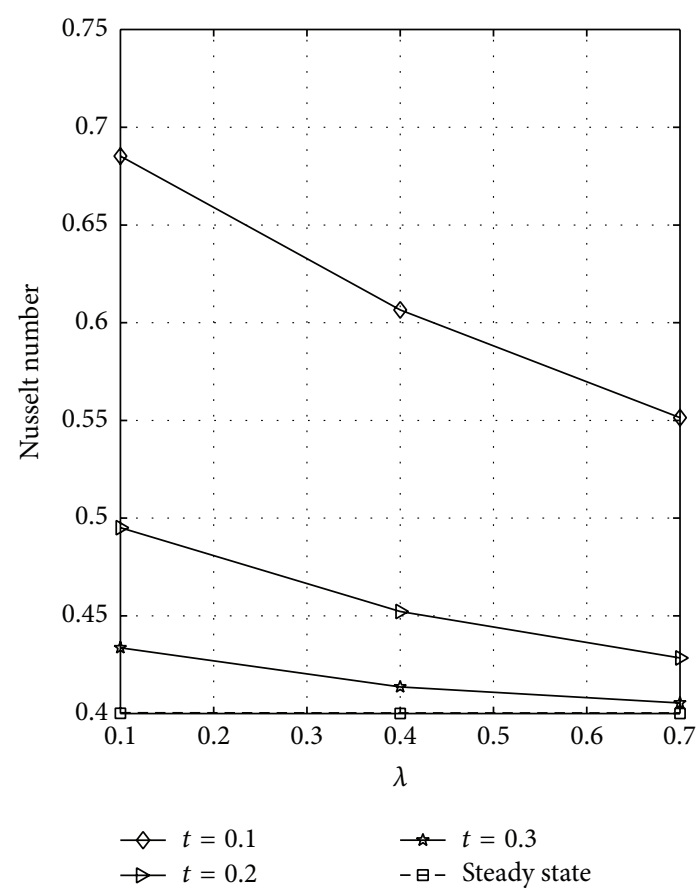

(a)

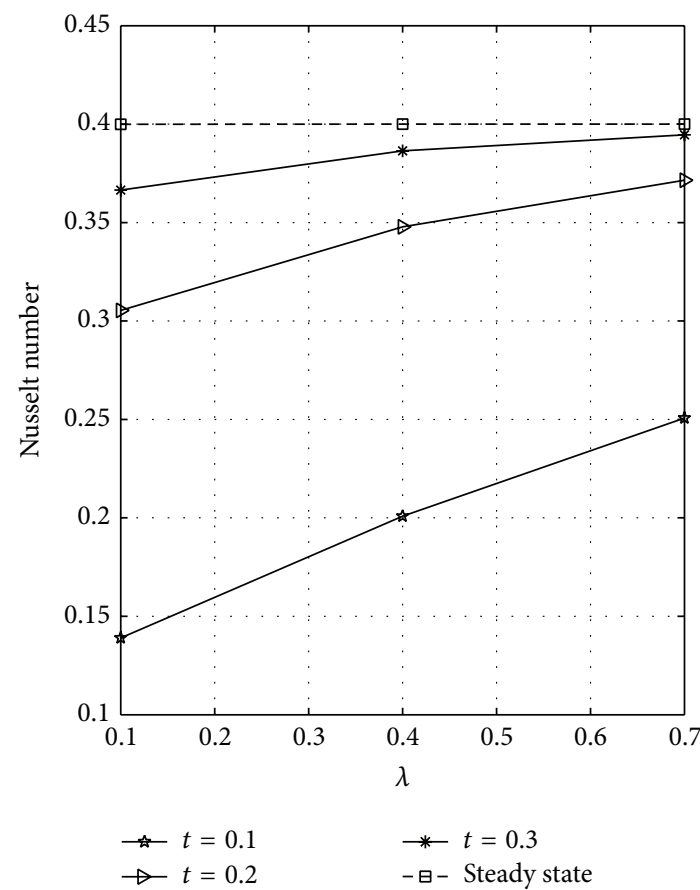

(b)

Figure 11: Variation of unsteady and steady-state Nusselt number with $\lambda, R=0$.

at the plate $y=0$ for asymmetric case. Figure 13(a) reflected that skin friction increases as $\lambda$ and $t$ increase until a steadystate condition is attained. Figure 13 (b) reveals that Nusselt number decreases with increasing $\lambda$ and $t$. It should be noted that the numerical values of skin friction at the plates $y=0$ and $y=1$ are the same for $R=1$ because a symmetric flow occurs for this case. Also, the Nusselt number at the plates $y=0$ and $y=1$ is the same for $R=1$.

The skin friction dependence on $M$ for varying values of $t$ at the plates $y=0$ and $y=1$ is displayed in Figures 14(a) and 14(b), respectively, for $R=0$. These figures reflected that skin friction decreases as $M$ and $t$ increase. Figures 15(a) and 15(b) are plotted to see the effects of $M$ on the skin friction at the plates $y=0$ and $y=1$ for $R=1$. From these figures, it is observed that skin friction decreases as $M$ and $t$ increase. Further, it is noted that Figures 15(a) and 15(b) are exactly the same. This is because a symmetric flow occurs for this case; that is, $R=1$.

\section{Conclusion}

The problem of unsteady as well as steady hydromagnetic natural convection flow of a viscous, incompressible, and electrically conducting fluid between two vertical walls having 


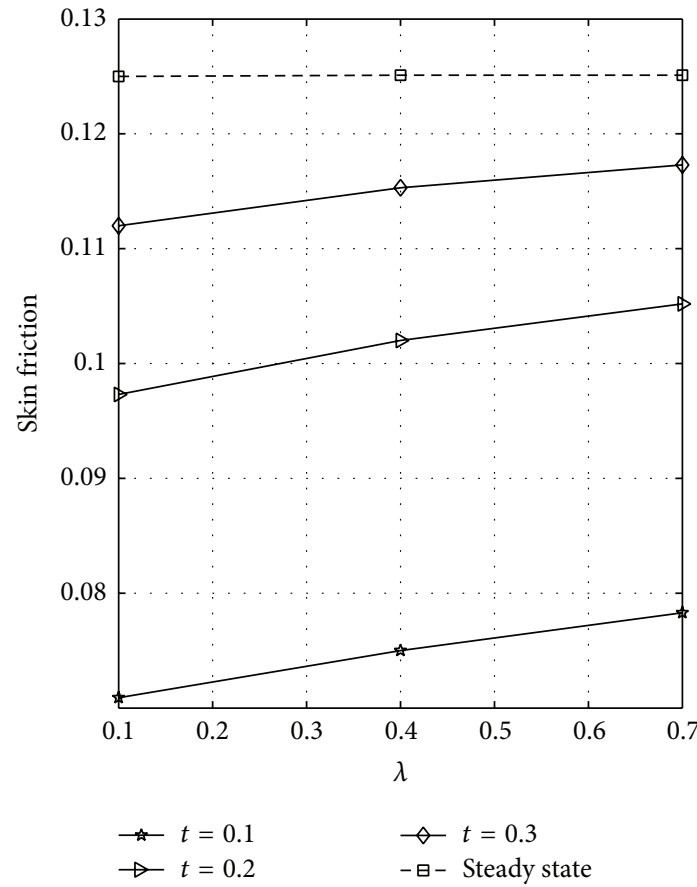

(a)

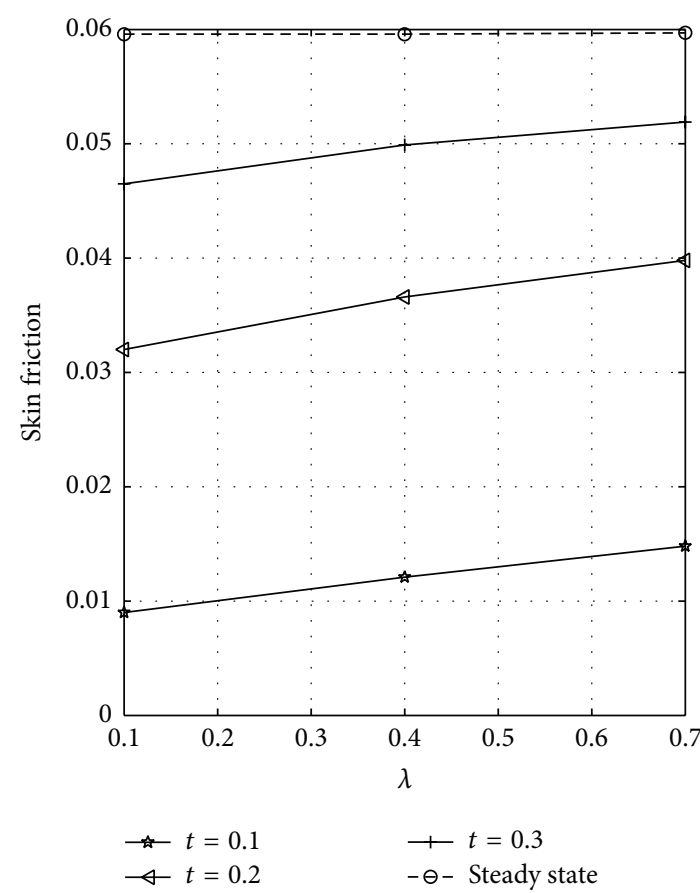

(b)

FIGURE 12: Variation of unsteady and steady-state skin friction with $\lambda, R=0$.

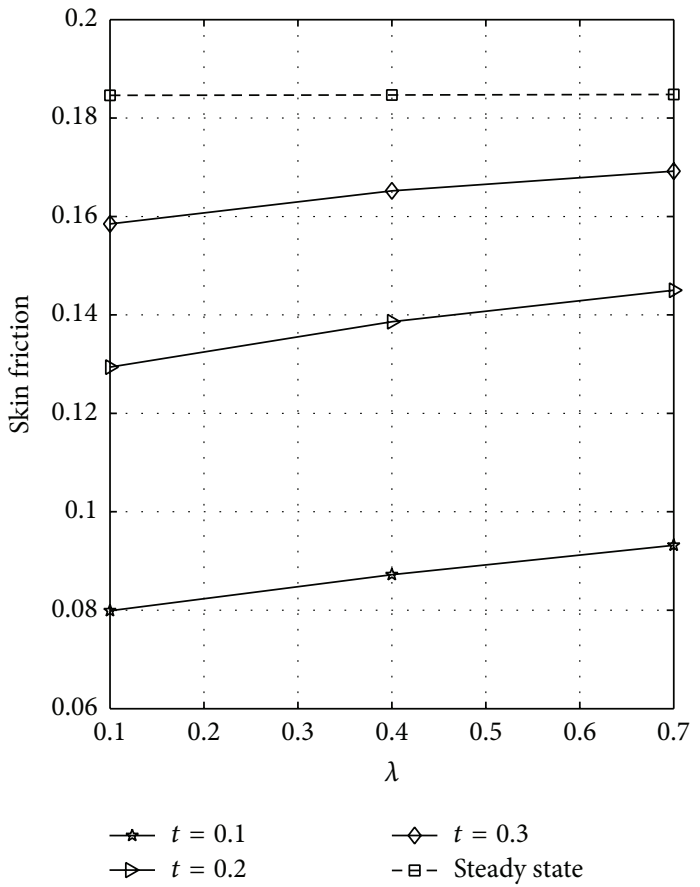

(a)

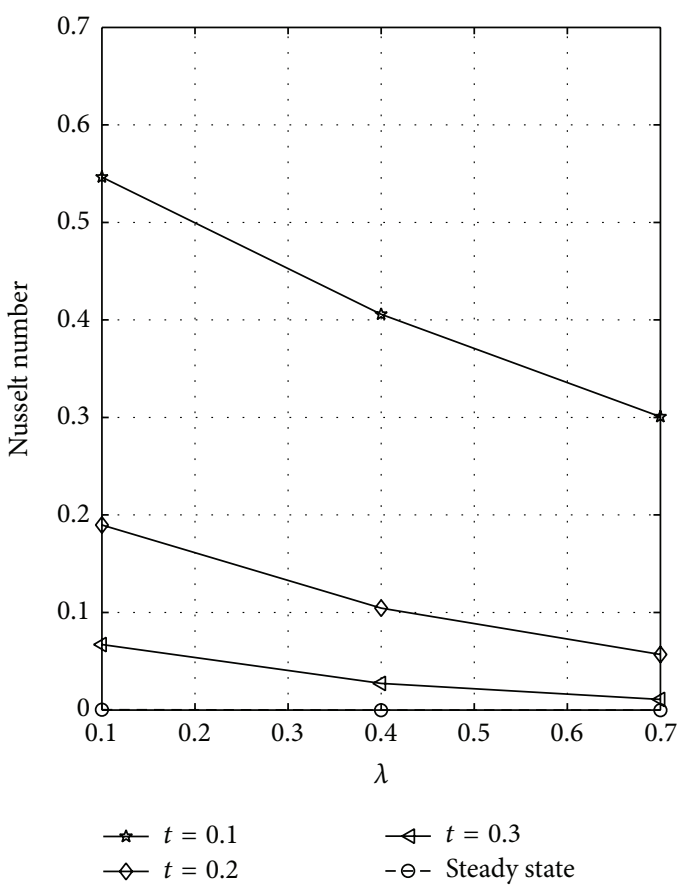

(b)

FIGURE 13: Variation of unsteady and steady-state skin friction and Nusselt number with $\lambda, R=1$. 


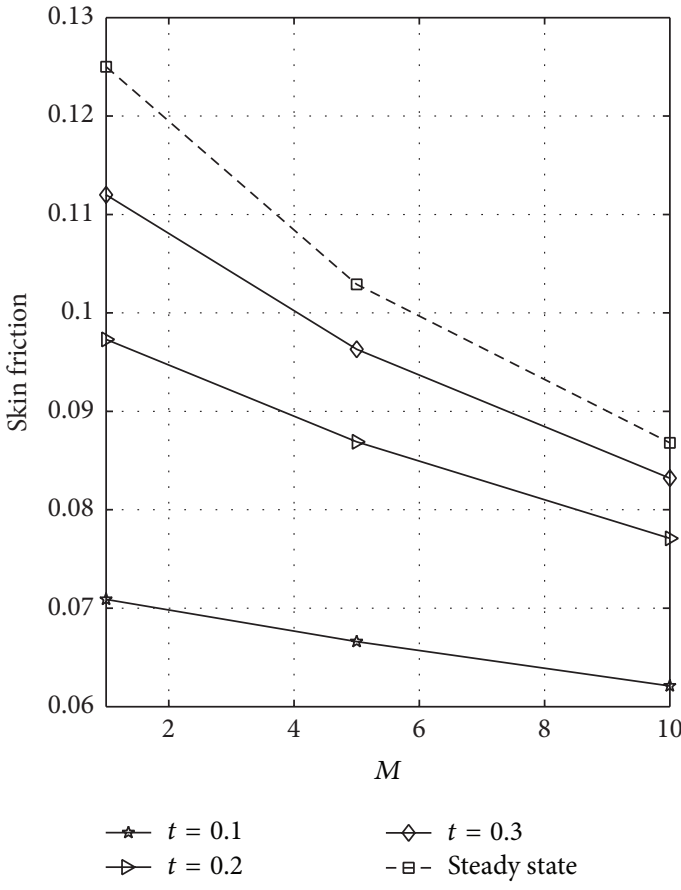

(a)

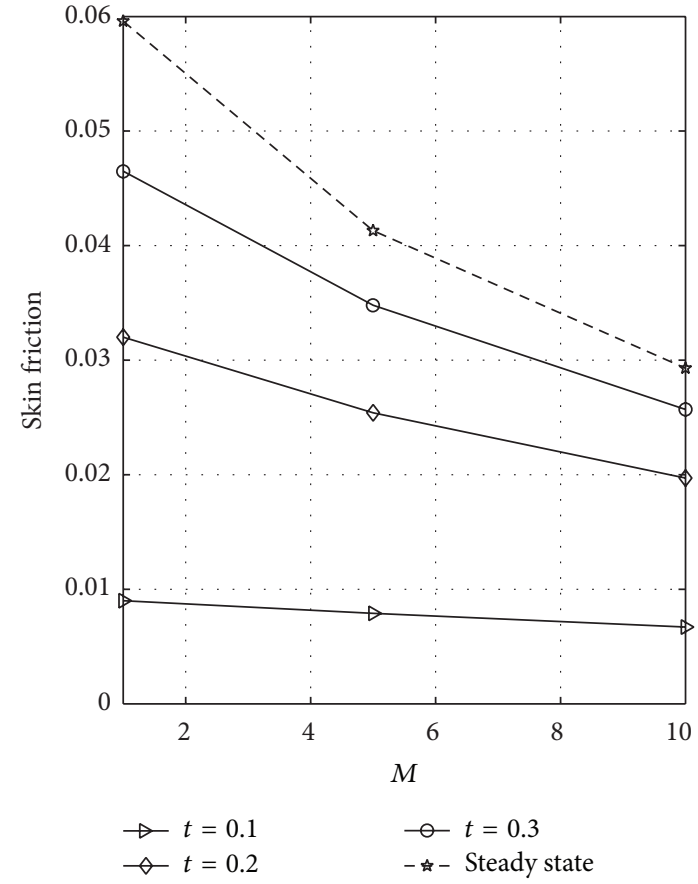

(b)

FIGURE 14: Variation of unsteady and steady-state skin friction with $M, R=0$.

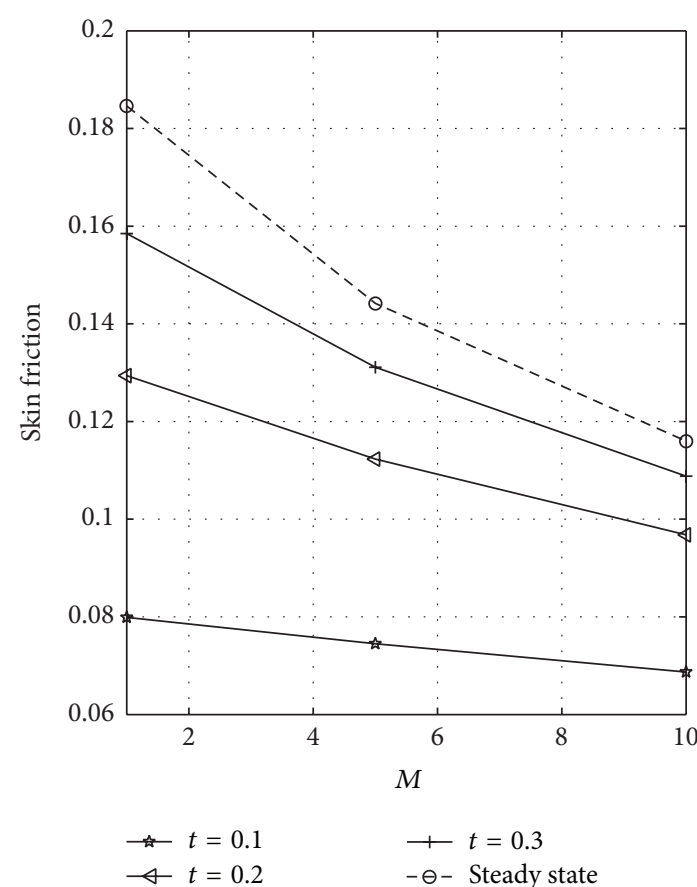

(a)

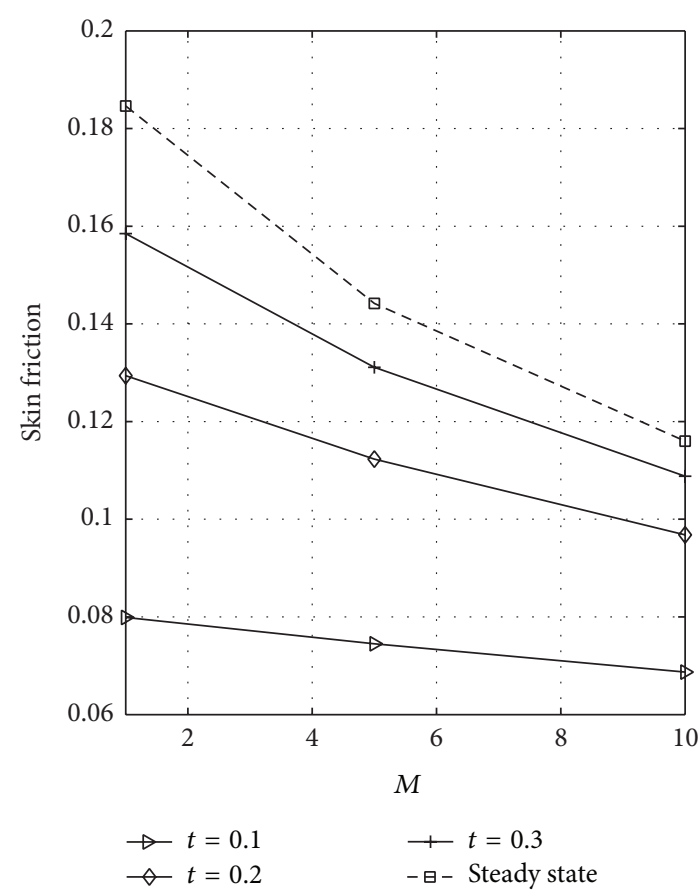

(b)

FIGURE 15: Variation of unsteady and steady-state skin friction with $M, R=1$.

asymmetric/symmetric temperatures on the walls in the presence of variable thermal conductivity and uniform applied magnetic field has been studied by obtaining approximated and numerical solutions of the governing equations, using perturbation series method and semi-implicit finite difference scheme. It is found that the fluid velocity and temperature increase with the increasing of variable thermal conductivity and nondimensional time for both asymmetric 
and symmetric heating, while magnetic parameter retards the motion of the fluid.

\section{Conflict of Interests}

The authors declare that there is no conflict of interests regarding the publication of this paper.

\section{References}

[1] J. Hartmann and F. Lazarus, "Hg-dynamics II: 'theory of laminar flow of electrically conductive liquids in a homogeneous magnetic field"' Matematisk-Fysiske Meddelelser, vol. 15, no. 7, 1937.

[2] R. M. Singer, “Transient magnetohydrodynamic flow and heat transfer," Zeitschrift für angewandte Mathematik und Physik, vol. 16, no. 4, pp. 483-494, 1965.

[3] G. S. Seth and R. N. Jana, "Unsteady hydromagnetic flow in a rotating channel with oscillating pressure gradient," Acta Mechanica, vol. 37, no. 1-2, pp. 29-41, 1980.

[4] G. S. Seth, R. N. Jana, and M. K. Maiti, "Unsteady hydromagnetic couette flow in a rotating system," International Journal of Engineering Science, vol. 20, no. 9, pp. 989-999, 1982.

[5] G. S. Seth and S. K. Ghosh, "Unsteady hydromagnetic flow in a rotating channel in the presence of inclined magnetic field," International Journal of Engineering Science, vol. 24, no. 7, pp. 1183-1193, 1986.

[6] P. Chandran, N. C. Sacheti, and A. K. Singh, "Effect of rotation on unsteady hydromagnetic Couette flow," Astrophysics and Space Science, vol. 202, no. 1, pp. 1-10, 1993.

[7] A. K. Singh, N. C. Sacheti, and P. Chandran, "Transient effects on magnetohydrodynamic couette flow with rotation: accelerated motion," International Journal of Engineering Science, vol. 32, no. 1, pp. 133-139, 1994.

[8] B. K. Jha and C. A. Apere, "Combined effect of hall and ion-slip currents on unsteady MHD couette flows in a rotating system," Journal of the Physical Society of Japan, vol. 79, Article ID 104401, 9 pages, 2010.

[9] B. K. Jha and C. A. Apere, "Magnetohydrodynamic free convective Couette flow with suction and injection," Journal of Heat Transfer, vol. 133, no. 9, Article ID 092501, 12 pages, 2011.

[10] N. C. Sacheti, P. Chandran, and A. K. Singh, "An exact solution for unsteady magnetohydrodynamic free convection flow with constant heat flux," International Communications in Heat and Mass Transfer, vol. 21, no. 1, pp. 131-142, 1994.

[11] M. A. Hossain, K. Khanafer, and K. Vafai, “The effect of radiation on free convection flow of fluid with variable viscosity from a porous vertical plate," International Journal of Thermal Sciences, vol. 40, no. 2, pp. 115-124, 2001.

[12] M. Arunachalam and N. R. Rajappa, "Forced convection in liquid metals with variable thermal conductivity and capacity," Acta Mechanica, vol. 31, no. 1-2, pp. 25-31, 1978.

[13] T. C. Chiam, "Heat transfer with variable conductivity in a stagnation-point flow towards a stretching sheet," International Communications in Heat and Mass Transfer, vol. 23, no. 2, pp. 239-248, 1996.

[14] T. C. Chiam, "Heat transfer in a fluid with variable thermal conductivity over a linearly stretching sheet," Acta Mechanica, vol. 129, no. 1-2, pp. 63-72, 1998.

[15] M. A. Seddeek and A. M. Salem, "Laminar mixed convection adjacent to vertical continuously stretching sheets with variable viscosity and variable thermal diffusivity," Heat and Mass Transfer, vol. 41, no. 12, pp. 1048-1055, 2005.

[16] M. M. Rahman, A. A. Mamun, M. A. Azim, and M. A. Alim, "Effects of temperature dependent thermal conductivity on magnetohydrodynamic free convection flow along a vertical flat plate with heat conduction," Nonlinear Analysis: Modelling and Control, vol. 13, pp. 513-524, 2008.

[17] P. R. Sharma and G. Singh, "Effects of variable thermal conductivity and heat source/sink on MHD flow near a stagnation point on a linearly stretching sheet," Journal of Applied Fluid Mechanics, vol. 2, no. 1, pp. 13-21, 2009.

[18] M. A. Alim, M. R. Karim, and M. M. Akand, "Heat generation effects on MHD natural convection flow along a vertical wavy surface with variable thermal conductivity," American Journal of Computational Mathematics, vol. 2, no. 1, pp. 42-50, 2012.

[19] M. G. Reddy, "Effects of thermophoresis, viscous dissipation and joule heating on steady MHD flow over an inclined radiative isothermal permeable surface with variable thermal conductivity," Journal of Applied Fluid Mechanics, vol. 7, no. 1, pp. 51-61, 2014.

[20] E. M. E. Elbarbary and N. S. Elgazery, "Chebyshev finite difference method for the effects of variable viscosity and variable thermal conductivity on heat transfer from moving surfaces with radiation," International Journal of Thermal Sciences, vol. 43, no. 9, pp. 889-899, 2004.

[21] A. K. Singh and T. Paul, "Transient natural convection between two vertical walls heated/cooled asymmetrically," International Journal of Applied Mechanics and Engineering, vol. 11, pp. 143154, 2006.

[22] J. Ettefagh, K. Vafai, and S. J. Kim, "Non-Darcian effects in openended cavities filled with a porous medium," Journal of Heat Transfer, vol. 113, no. 3, pp. 747-756, 1991.

[23] B. Alazmi and K. Vafai, "Analysis of variants within the porous media transport models," Journal of Heat Transfer, vol. 122, no. 2, pp. 303-326, 2000. 

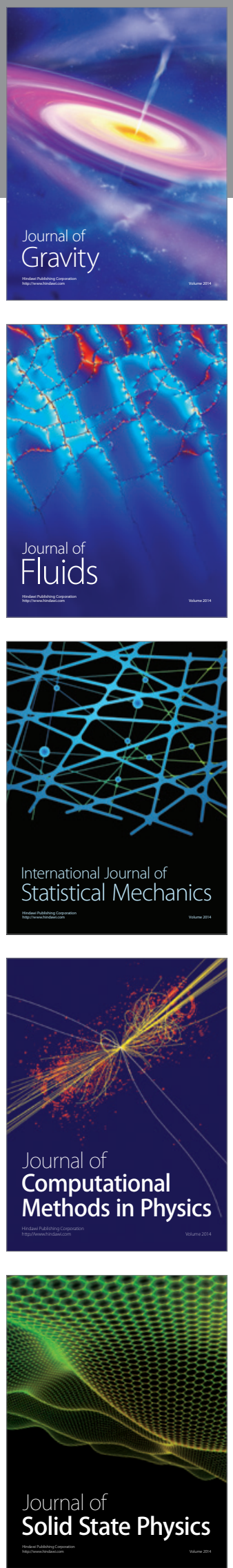

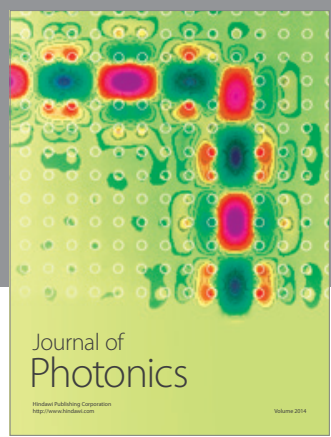

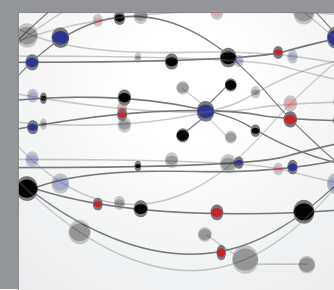

The Scientific World Journal

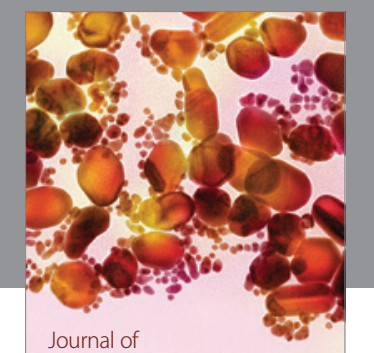

Soft Matter
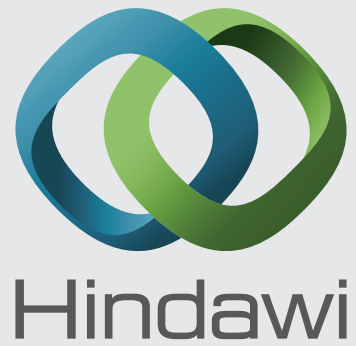

Submit your manuscripts at

http://www.hindawi.com
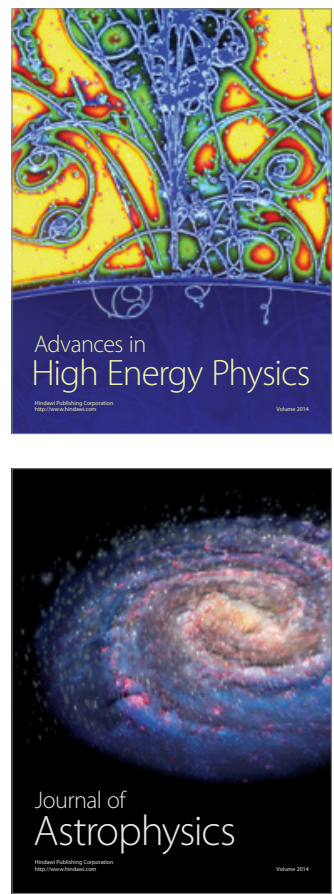
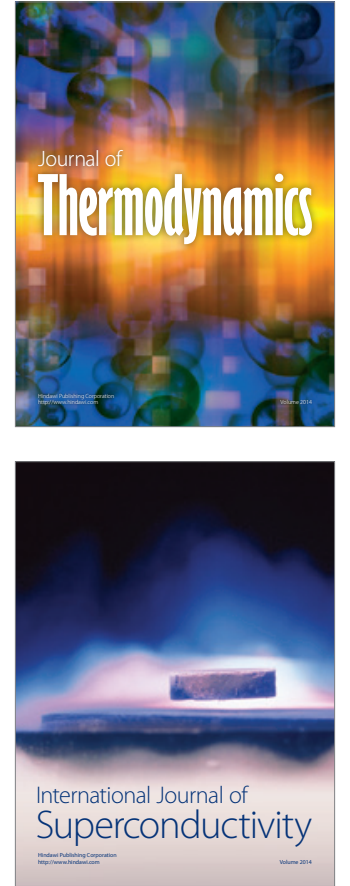
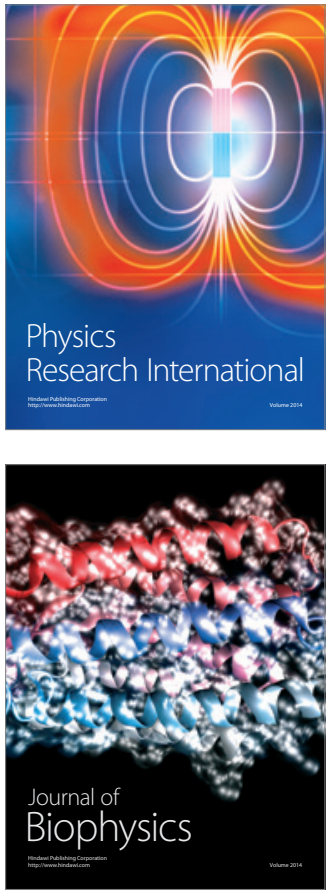
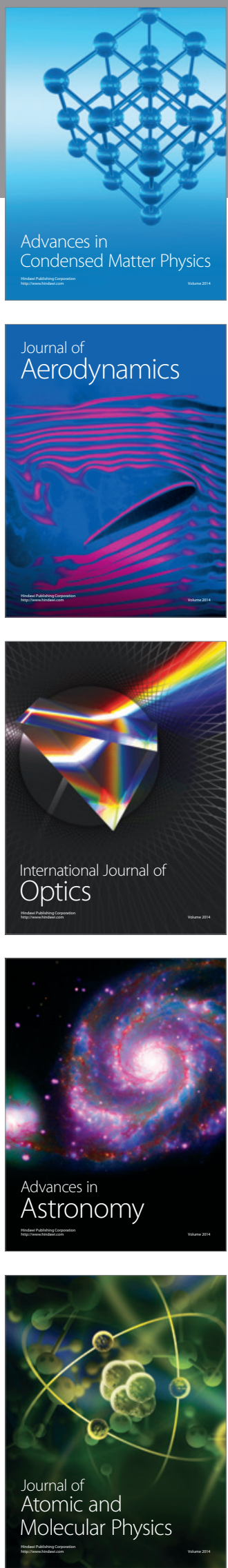